\title{
miR-338-3p mediates gluconeogenesis via targeting of PP4R1 in hepatocytes
}

\author{
SHUYUE WANG ${ }^{1,2^{*}}$, LINFANG LI $^{1 *}$, XIEHUI CHEN ${ }^{3 *}$, XIUQING HUANG $^{1}$, JIN LIU $^{4}$, XUELIN SUN $^{1}$, \\ YANG ZHANG $^{1}$, TAO SHEN ${ }^{1}$, JUN GUO ${ }^{1}$, YONG MAN ${ }^{1}$, WEIQING TANG ${ }^{1}$, LIN DOU $^{1}$ and JIAN LI ${ }^{1}$ \\ ${ }^{1}$ The MOH Key Laboratory of Geriatrics, Beijing Hospital, National Center of Gerontology, Beijing 100730; \\ ${ }^{2}$ National Engineering Laboratory for Druggable Gene and Protein Screening, Northeast Normal University, Changchun, \\ Jilin 130117; ${ }^{3}$ Department of Geriatrics Cardiovascular Medicine, Shenzhen Sun Yat-Sen Cardiovascular Hospital, \\ Shenzhen, Guangdong 518112; ${ }^{4}$ College of Life Sciences, Beijing Normal University, Beijing 100875, P.R. China
}

Received January 15, 2018; Accepted July 13, 2018

DOI: $10.3892 / \mathrm{mmr} .2018 .9400$

\begin{abstract}
Hyperglycaemia is a characteristic of type 2 diabetes. In hepatocytes, impaired insulin sensitivity leads to increased gluconeogenesis and decreased glycogenesis. MicroRNA (miR)-338-3p is associated with tumour necrosis factor (TNF)- $\alpha$-induced suppression of hepatic glycogenesis via regulation of protein phosphatase 4 regulatory subunit 1 (PP4R1). However, the effect of miR-338-3p on gluconeogenesis in hepatocytes remains unknown. In a previous study, it was demonstrated that miR-338-3p is downregulated in the livers of mice and in mouse HEPA1-6 hepatocytes following treatment with TNF- $\alpha$. In the present study, the effect of miR-338-3p on TNF- $\alpha$-induced gluconeogenesis in hepatocytes was investigated. The levels of phosphorylated-FOXO1/FOXO1, phosphoenolpyruvate carboxykinase (PEPCK), peroxisome proliferator-activated receptor $\gamma$ coactivator (PGC-1 $\alpha$ ) and glucose-6-phosphatase (G6Pase) were measured by western blotting. The mRNA levels of PEPCK, PGC-1 $\alpha$ and G6Pase were determined by quantitative polymerase chain reaction. Pyruvate tolerance testing was used to determine the gluconeogenesis of mouse livers. The results demonstrated that treatment with TNF- $\alpha$ resulted in increased levels of gluconeogenesis in the livers of mice and decreased miR-338-3p expression levels in HEPA1-6 cells. Overexpression of miR-338-3p reversed TNF- $\alpha$-induced glucose production via enhancement of
\end{abstract}

Correspondence to: Dr Lin Dou or Dr Jian Li, The MOH Key Laboratory of Geriatrics, Beijing Hospital, National Center of Gerontology, 1 Dahua Road, Beijing 100730, P.R. China

E-mail: doulin4623@bjhmoh.cn

E-mail: 1ijian@bjhmoh.cn

*Contributed equally

Key words: microRNA-338-3p, gluconeogenesis, protein phosphatase 4 regulatory subunit 1 , forkhead box O1, peroxisome proliferator-activated receptor $\gamma$ coactivator- $1 \alpha$, phosphoenolpyruvate carboxykinase, glucose-6-phosphatase phosphorylated forkhead box O1 levels and downregulation of the expression levels of genes associated with gluconeogenesis, including peroxisome proliferator-activated receptor $\gamma$ coactivator- $1 \alpha$, phosphoenolpyruvate carboxykinase and glucose-6-phosphatase. However, inhibition of miR-338-3p expression was revealed to enhance gluconeogenesis in the livers of mice and in HEPA1-6 cells. Furthermore, downregulation of PP4R1 was revealed to attenuate the effect on glucose production following treatment with miR-338-3p inhibitors. In conclusion, the results of the present study revealed that miR-338-3p may be involved in TNF- $\alpha$-mediated gluconeogenesis via targeting of PP4R1 in hepatocytes.

\section{Introduction}

The incidence of obesity and aging has been associated with Type 2 diabetes, a disease of increasing importance in social health due to its prevalence. (1) Type 2 diabetes affects the homeostasis of glucose metabolism and leads to serious complications, including diabetic cardiomyopathy and diabetic nephropathy (2). At present, a cure for type 2 diabetes is unavailable as the underlying mechanisms of the pathogenesis of type 2 diabetes is not completely understood (3). Hyperglycaemia is characteristic of type 2 diabetes (4). Hepatic insulin resistance is a predominant cause of type 2 diabetes. In the liver, glucose metabolism homeostasis is regulated via two processes, gluconeogenesis and glycogenesis (5). Impaired insulin sensitivity in the liver leads to increased gluconeogenesis and decreased glycogenesis (6).

In the liver cells, insulin can decrease glucose production and increase uptake of blood glucose into glycogen via the phosphatidylinositol-4,5-bisphosphate 3-kinase (PI3K)/protein kinase B (AKT) pathway (7). Additionally, activated AKT promotes the phosphorylation of forkhead box O1 (FOXO1), which can translocate into nuclear and inhibit the expression of gluconeogenetic enzymes and gluconeogenesis (8). Peroxisome proliferator-activated receptor $\gamma$ coactivator (PGC-1 $\alpha$ ) is another important regulator of gluconeogenesis in liver cells. PGC-1 $\alpha$ can co-activate FOXO1 and enhance the transcription of phosphoenolpyruvate carboxykinase (PEPCK) and glucose-6-phosphatase (G6Pase). PGC-1 $\alpha$, 
PEPCK and G6Pase are key regulators of gluconeogenesis in liver cells (9).

MicroRNAs (miRs) are a group of small, non-coding RNAs. miRs negatively modulate gene expression by directly binding to a partially complementary sequence in the 3 '-untranslated region (UTR) of their target mRNA $(10,11)$. Tumour necrosis factor (TNF)- $\alpha$ is an important mediator of insulin resistance that functions by inhibiting the PI3K/AKT pathway. Furthermore, in vitro and in vivo studies have suggested that TNF- $\alpha$ treatment leads to increased gluconeogenesis and decreased glycogenesis in hepatocytes (12). Our previous study demonstrated that miR-338-3p overexpression restores impaired glycogenesis induced by TNF- $\alpha$ by directly inhibiting protein phosphatase 4 regulatory subunit 1 (PP4R1) expression (13). However, the association between miR-338-3p and increased levels of gluconeogenesis induced by TNF- $\alpha$ remains unknown. In the present study, the effects of miR-338-3p on gluconeogenesis and the associated underlying mechanisms were investigated. The results suggested that miR-338-3p may have an important role in TNF- $\alpha$-induced gluconeogenesis via targeting of PP4R1.

\section{Materials and methods}

Animals. In order to establish a TNF- $\alpha$-induced insulin resistance model, 16 -week-old male $\mathrm{C} 57 \mathrm{BL} / 6 \mathrm{~J}$ mice $(\mathrm{n}=5$; 30 g, Beijing HFK Bioscience Co., Ltd., Beijing, China) were fed with a standard laboratory diet and injected with $15 \mu \mathrm{g} / \mathrm{ml}$ TNF- $\alpha$ via Alzet osmotic pumps (DURECT Corporation, Cupertino, CA, USA) for 2 weeks continuously at an infusion rate of $0.5 \mu \mathrm{l} / \mathrm{h}$. For the control group, 16-week-old male C57BL/6J mice $(n=5)$ were injected with $0.9 \% \mathrm{NaCl}$ via Alzet osmotic pumps for 2 weeks at the aforementioned infusion rate. Following fasting for $8 \mathrm{~h}$, mice were subsequently anaesthetized via intrapertioneal injection with sodium pentobarbital (40 mg/kg body weight), and liver samples were then collected. All mice were housed in an experimental animal facility of Beijing Normal University (Beijing, China) at a temperature of $20-24^{\circ} \mathrm{C}$ and humidity-controlled (45-55\%) environment. A $12 \mathrm{~h}$ light/dark cycle was maintained in the animal housing rooms. The food and water were supplied for all days. All animal experiments performed in the present study were approved by the Animal Use and Care Committee of Beijing Normal University and were performed in accordance with the Guide for Care and Use of Laboratory Animals (14).

Adenoviral vector injection. For animal experiments, 6 mice were used in each group. To investigate the effect of miR-338-3p on TNF- $\alpha$-induced gluconeogenesis, after 7 days following the implantation of the TNF- $\alpha$ pumps, 6 C57BL/6J mice were injected with adenoviral vectors expressing miR-338-3p mimics (Ad-338m) $\left(2 \times 10^{9}\right.$ plaque-forming unit/25 g body weight). A total of $6 \mathrm{C} 57 \mathrm{BL} / 6 \mathrm{~J}$ mice were injected with adenoviral vectors expressing negative control microRNA (Ad-NC) $\left(2 \times 10^{9}\right.$ plaque-forming unit/25 g body weight). In addition, adenoviral vectors expressing miR-338-3p inhibitors (Ad-338i) or negative control inhibitors (Ad-NCI) were injected into $6 \mathrm{C} 57 \mathrm{BL} / 6 \mathrm{~J}$ mice $\left(2 \times 10^{9}\right.$ plaque-forming unit/25 g body weight). All the adenoviral vectors, including Ad-338m, Ad-NC, Ad-338i and Ad-NCI were purchased from
Shanghai GeneChem Co., Ltd. (Shanghai, China). After 7 days following injection, mice were used for following experiments. The sequences employed in the present study were as follows (5'-3'): miR-338-3p mimic, UCCAGCAUCAGUGAUUUUGU and negative control mimics, UUCUCCGAACGUGUCACG UTT; miR-338-3p inhibitor, CAACAAAAUCACUGAUGC UGGA and negative control inhibitors, CAGUACUUUUGU GUAGUACAA.

Pyruvate tolerance testing. After 7 days following injection, pyruvate tolerance analysis was performed. Following fasting for $14 \mathrm{~h}$, mice were intraperitoneally injected with pyruvate $(2 \mathrm{~g} / \mathrm{kg}$ of body weight) in order to perform pyruvate tolerance testing. Blood glucose levels were measured using an ACCU-CHEK Advantage glucometer (Roche Diagnostics $\mathrm{GmbH}$, Mannheim, Germany) at specific time intervals.

Cell culture. HEPA1-6 murine liver cells (American Type Culture Collection, Manassas, VA, USA) were cultured in low-glucose Dulbecco's modified Eagle's medium (DMEM; Invitrogen; Thermo Fisher Scientific, Inc., Waltham, MA, USA) supplemented with $10 \%$ fetal serum (HyClone; GE Healthcare Life Sciences, Logan, UT, USA), 100 units/ml penicillin (Invitrogen; Thermo Fisher Scientific, Inc.) and $0.1 \mathrm{mg} / \mathrm{ml}$ streptomycin (Hyclone; GE Healthcare Life Sciences) at $37^{\circ} \mathrm{C}$ with humidified air and $5 \% \mathrm{CO}_{2}$. In order to determine the effect of TNF- $\alpha$ on gluconeogenesis, the HEPA1- 6 cells were treated with $10 \mathrm{ng} / \mathrm{ml} \mathrm{TNF}-\alpha$ for $24 \mathrm{~h}$ at $37^{\circ} \mathrm{C}$ with humidified air and $5 \% \mathrm{CO}_{2}$.

Transfection. miR-338-3p mimic and inhibitor sequences used for transfection were as follows (5'-3'): miR-338-3p mimic sense, UCCAGCAUCAGUGAUUUUGUUG and antisense, ACAAAAUCACUGAUGCUGGAUU; NC sense, UUC UCCGAACGUGUCACGUTT and antisense, ACGUGACAC GUUCGGAGAATT; miR-338-3p inhibitor, CAACAAAAU CACUGAUGCUGGA; NCI, CAGUACUUUUGUGUAGUA CAA. All miR oligos were purchased from Genepharm, Inc. (Sunnyvale, CA, USA). A day before transfection, 3.6x10 5 cells were seeded in each well of 6-well plate in $1.6 \mathrm{ml}$ DMEM medium. miR-338-3p mimic and inhibitor (3.75 $\mu 1,20 \mu \mathrm{M})$ were transfected into HEPA1-6 by using HiPerFect transfection reagent (Qiagen, Inc., Valencia, CA, USA). Subsequent experimentation was performed $48 \mathrm{~h}$ following transfection.

The sequences of PP4R1-specific small interfering (siRNAs) used for transfection were as follows (5'-3'): PP4R1 sense, GAACCAACTGTGAGAGCGGA; and antisense, TGG GGCAGTCAGGTCTATGA. All siRNA oligos were purchased from Genepharm, Inc. A day before transfection, $3.6 \times 10^{5}$ cells were planted in each well of 6 -well plate in $1.6 \mathrm{ml}$ DMEM. PP4R1-specific siRNAs $(3.75 \mu \mathrm{l}, 20 \mu \mathrm{M})$ were transfected into HEPA1-6 cells using HiPerFect transfection reagent (Qiagen, Inc.). Subsequent assays were performed $48 \mathrm{~h}$ following transfection.

Reverse transcription-quantitative polymerase chain reaction (RT-qPCR). Total RNA of HEPA1-6 cells and mouse liver cells was extracted using TRIzol (Invitrogen; Thermo Fisher Scientific, Inc.). A total of $1 \mu \mathrm{g}$ RNA was reverse transcribed into cDNA using the EasyScript First-strand cDNA Synthesis 
kit (Beijing Transgen Biotech Co., Ltd., Beijing, China). The procedures of RT were described as follow: $70^{\circ} \mathrm{C}$ for $10 \mathrm{~min}$, $42^{\circ} \mathrm{C}$ for $60 \mathrm{~min}, 95^{\circ} \mathrm{C}$ for $5 \mathrm{~min}$ and $4^{\circ} \mathrm{C}$ hold. Expression levels of PGC-1 $\alpha$, PEPCK and G6Pase were determined via qPCR using the SYBR Green II kit (Takara Biotechnology Co., Ltd., Dalian, China) in accordance with the manufacturer's protocol. cDNA $(1 \mu \mathrm{g}), 10 \mu \mathrm{l}$ SYBR-Green mixture, $0.8 \mu \mathrm{l}$ primers

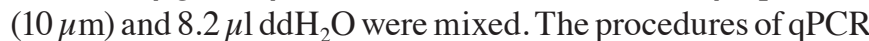
were as follows: $95^{\circ} \mathrm{C}$ for $30 \mathrm{sec}$, followed by 40 cycles at $95^{\circ} \mathrm{C}$ for $5 \mathrm{sec}$ and $60^{\circ} \mathrm{C}$ for $20 \mathrm{sec}$. The sequences of primers used for PCR were as follows (5'-3'): G6Pase forward, TCTGTC CCGGATCTACCTTG and reverse, GTAGAATCCAAGCGC GAAA; PEPCK forward, TCTGAGATCTCTGATCCAGAC $\mathrm{C}$ and reverse, GAAGTCCAGACCGTTATGCAGC; PGC- $1 \alpha$ forward, GCCTATGAGCACGAAAGGC and reverse, TCA CACGGCGCTCTTCAATT; 18s forward, GGAAGGGCA CCACCAGGAGT and reverse, TGCAGCCCCGGACATCTA $A G$. The gene expression level was calculated using $2^{-\Delta \Delta C q}$ method (15).

Western blotting. Total protein from liver and cells was extracted with radioimmunoprecipitation (Beijing Solarbio Science \& Technology Co., Ltd., Beijing, China). And the concentration of protein sample was measure by BCA kit (Sigma-Aldrich; Merck KGaA, Darmstadt, Germany). Cell protein samples $(15 \mu \mathrm{g})$ were separated via 10\% SDS-PAGE, transferred to polyvinylidene fluoride membranes (EMD Millipore, Billerica, MA, USA) and then blocked using $8 \%$ non-fat dry milk for $2 \mathrm{~h}$ at room temperature. Following this, the membranes incubated with 1:1,000 diluted primary antibodies for $12 \mathrm{~h}$ at $4^{\circ} \mathrm{C}$. The blots were then incubated with 1:5,000 diluted horseradish peroxidase-conjugated anti-IgG (cat. no. 7074; Cell Signaling Technology, Inc. Danvers, MA, USA) for $2 \mathrm{~h}$ at room temperature. Proteins were then visualized using an enhanced chemiluminescent kit (EMD Millipore). Antibodies against FOXO1 (cat. no. 2880), phosphorylated FOXO1 (cat. no. 9461), PEPCK (cat. no. 6924) and GAPDH (cat. no. 5174) were purchased from Cell Signaling Technology, Inc. Antibodies against PGC-1 $\alpha$ (ab54481) and G6Pase (ab83690) were purchased from Abcam (Cambridge, UK).

Luciferase reporter assay. TargetScan (http://www. targetscan.org/), Pictar (http://www.pictar.org/) and miRanda (http://microrna.org/) databases were used to predict that PP4R1 was a target of miR-338-3p. The miR-338-3p binding site with in the PP4R1 3'-UTR was verified and in previously study (13). The PP4R1 3'-UTR fragment, including the miR-338-3p binding site was cloned from mouse liver cells and inserted into pmirGLO vector (Promega Corporation, Madison, WI, USA). A day prior to transfection, HEPA1-6 cells were seeded at $3.6 \times 10^{5}$ cells per well of 6 -well plate in $1.6 \mathrm{ml}$ DMEM. pmirGLO-3'UTR PP4R1 with miR-338-3p mimic or inhibitor $(3.75 \mu 1,20 \mu \mathrm{M})$ were transfected into HEPA1-6 by using HiPerFect transfection reagent. The subsequent assays were performed after $48 \mathrm{~h}$ post-transfection. Luciferase activity was analyzed by using the Dual-Glo Luciferase assay system (Promega Corporation). Luciferase activity was normalized to Renilla luciferase activity. All the assays were repeat for 4 times.
Glucose production assay. After $24 \mathrm{~h}$ of TNF- $\alpha$ treatment, a glucose production assay was performed. Cells were washed five times with PBS and then stimulated with $10 \mathrm{ng} / \mathrm{ml}$ TNF- $\alpha, 2 \mathrm{mmol} / 1$ sodium pyruvate and $20 \mathrm{mmol} / 1$ sodium lactate in glucose- and serum-free DMEM medium for $18 \mathrm{~h}$. The glucose concentration in the medium was subsequently determined using a glucose assay kit (Sigma-Aldrich; Merck $\mathrm{KGaA}$, Darmstadt, Germany) and subsequently normalized to the previously determined total protein content via a BCA kit (Sigma-Aldrich; Merck KGaA) in whole cell extracts.

Statistical analysis. All data are presented as the mean \pm standard error of the mean. The two-tailed unpaired Student's t-test was used for comparisons between two groups. In addition, One-way analysis of variance followed by Tukey's post hoc test was used for comparisons between more than two groups. Statistical analyses were performed using SPSS 3.0 software (SPSS, Inc., Chicago, IL, USA). P $<0.05$ was considered to indicate a statistically significant difference. All the experiments were repeated for four times.

\section{Results}

TNF- $\alpha$ treatment induces gluconeogenesis in mouse livers and HEPA1-6 cells. In our previous study, it was demonstrated that treatment with TNF- $\alpha$ decreased miR-338-3p expression levels and suppressed the activation of the AKT/GSK pathway in the livers of mice (13). In the present study, treatment with TNF- $\alpha$ significantly decreased the level of p-FOXO1/FOXO1 and significantly increased the protein and mRNA expression levels of genes associated with gluconeogenesis in the livers of mice, including PGC-1 $\alpha$, G6Pase and PEPCK (Fig. 1A and B). Consistently, treatment of $10 \mathrm{ng} / \mathrm{ml} \mathrm{TNF-} \alpha$ enhanced glucose production in HEPA1-6 cells (Fig. 1C). Furthermore, in HEPA1- 6 cells following treatment with $10 \mathrm{ng} / \mathrm{ml} \mathrm{TNF-} \alpha$ for $24 \mathrm{~h}$, the levels of p-FOXO1/FOXO1 were reduced, and the protein and mRNA expression of PGC-1 $\alpha$, G6Pase and PEPCK were increased (Fig. 1D and E).

Overexpression of $m i R-338-3 p$ attenuates $T N F-\alpha$-induced gluconeogenesis. To determine the importance of miR-338-3p in the regultion of gluconeogenesis, an adenovirus expressing miR-338-3p mimic was injected into TNF- $\alpha$-treated mice via the tail vein for 7 days. The results revealed that levels of p-FOXO1/FOXO1 were significantly increased, whereas mRNA and protein expression levels of PGC-1 $\alpha$, G6Pase and PEPCK were significantly decreased, in the livers of mice injected with Ad-338m compared with the control group accompanied by ameliorated pyruvate tolerance (Fig. 2A-C). In HEPA1-6 cells, transfection with miR-338-3p mimics was demonstrated to significantly attenuate $\mathrm{TNF}-\alpha$-induced glucose production (Fig. 2D). In addition, upregulation of miR-338-3p was revealed to significantly enhanced levels of p-FOXO1/FOXO1, and significantly decreased the expression levels of PGC-1 $\alpha$, G6Pase and PEPCK, compared with the NC group (Fig. 2E and F). In conclusion, these results suggested that miR-338-3p may have an important role in TNF- $\alpha$-induced gluconeogenesis.

Treatment with miR-338-3p inhibitors increases glucose production in hepatocytes. The effect of treatment with 

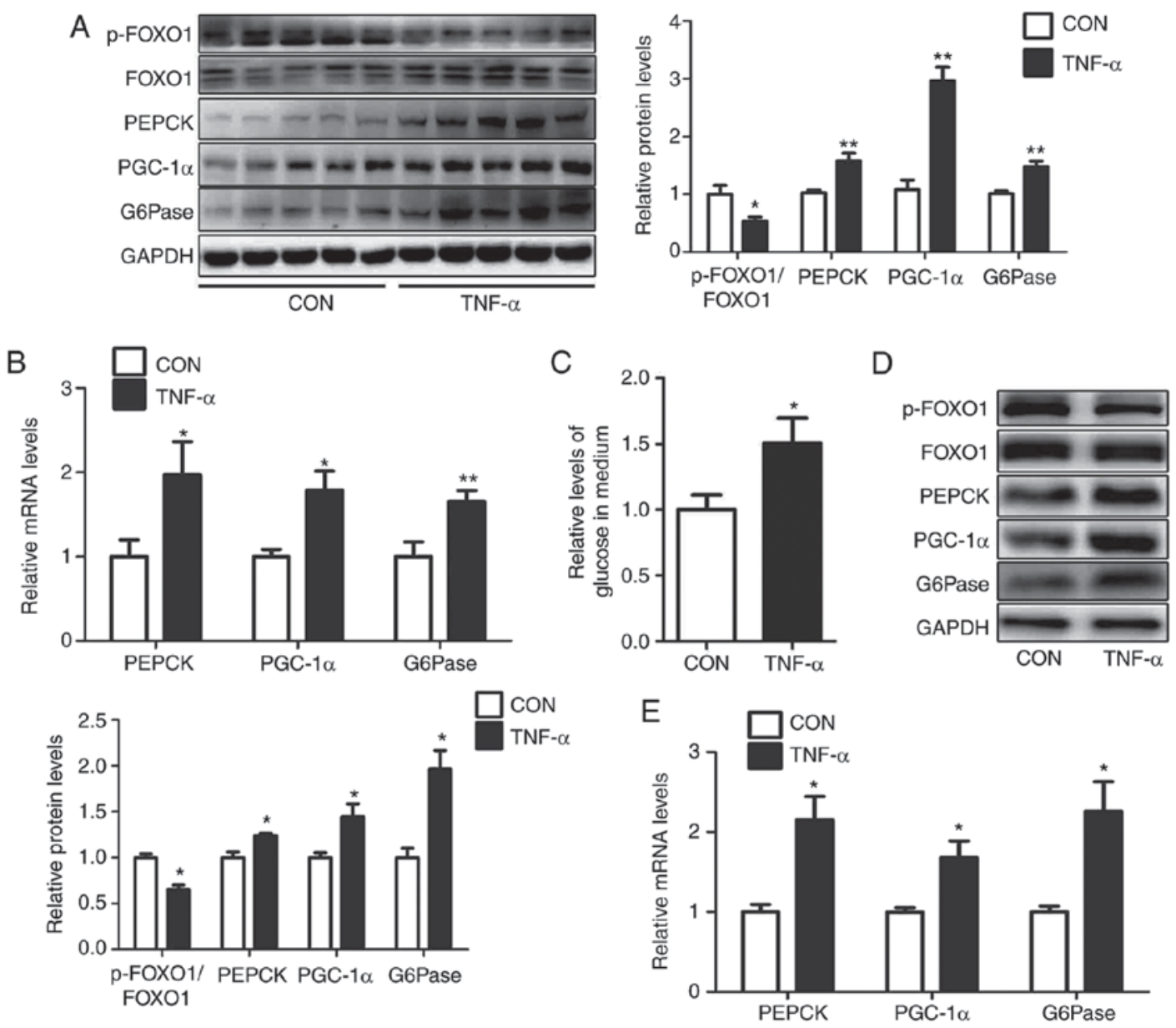

Figure 1. TNF- $\alpha$ treatment induces gluconeogenesis in mouse livers and HEPA1-6 hepatocytes. Levels of p-FOXO1/FOXO1, PEPCK, PGC-1 $\alpha$ and G6Pase in the livers of TNF- $\alpha$-treated mice were analyzed via (A) western blot and (B) reverse transcription-quantitative polymerase chain reaction. (C) Relative levels of glucose in culture medium were determined, as well as (D) protein and (E) mRNA levels of p-FOXO1/FOXO1, PEPCK, PGC-1 $\alpha$ and G6Pase, in HEPA1-6 cells treated with TNF- $\alpha$ for $24 \mathrm{~h}$. Data are presented as the mean \pm standard error. For animal experiments, 5 mice were used for each group. For cell experiments, $\mathrm{n}=4$ independent experiments. ${ }^{*} \mathrm{P}<0.05$ and ${ }^{* *} \mathrm{P}<0.01$ vs. control. p-, phosphorylated; FOXO1, forkhead box O1; PEPCK, phosphoenolpyruvate carboxykinase; G6Pase, glucose-6-phosphatase; PGC-1 $\alpha$, peroxisome proliferator-activated receptor $\gamma$ coactivator- $1 \alpha$; TNF- $\alpha$, tumor necrosis factor- $\alpha$; CON, control.

miR-338-3p inhibitors on glucose production in hepatocytes was investigated in the present study. An adenovirus expressing miR-338-3p inhibitor (Ad-338i) was injected into mice via the tail vein over 7 days. Inhibition of miR-338-3p decreased the levels of p-FOXO1/FOXO1 in livers of mice; the protein and mRNA expression levels of PGC-1 $\alpha$, G6Pase and PEPCK were increased in the liver, and pyruvate tolerance was impaired in mice injected with Ad-338i (Fig. 3A-C). Furthermore, HEPA1-6 hepatocytes transfected with a miR-338-3p inhibitor exhibited significantly reduced levels of p-FOXO1, increased levels of glucose production, and increased mRNA and protein expression of PGC-1 $\alpha$, G6Pase and PEPCK, compared with the NC group (Fig. 3D-F). These results suggested that miR-338-3p may regulate glucose production via the AKT/FOXO1 pathway.

miR-338-3p is involved in TNF- $\alpha$-mediated gluconeogenesis via targeting of PP4R1. In our previous study, PP4R1 was revealed to represent a target gene of miR-338-3p. The 3'-UTR of PP4R 1 was cloned and luciferase reporter vectors were subsequently constructed (13). The relative luciferase activity was significantly decreased following transfection with miR-338-3p mimics (Fig. 4A); however, relative luciferase activity was not markedly affected following transfection with miR-338-3p inhibitors (Fig. 4B). To verify whether miR-338-3p regulates glucose production via targeting of PP4R1, miR-338-3p inhibitors and PP4R1 siRNA were co-transfected into HEPA1-6 cells. The results revealed that downregulation of PP4R1 significantly attenuated miR-338-3p-induced increased levels of glucose production (Fig. 4C), and expression levels of genes associated with gluconeogenesis. However, transfection of PP4R1 siRNA reversed the effect of miR-338-3p inhibitor on the level of p-FOXO1/FOXO1 (Fig. 4D and E).

Furthermore, in order to determine whether PP4R1 is involved in the regulation of glucose production by TNF- $\alpha$, PP4R1 siRNA was transfected into HEPA1-6 cells for $48 \mathrm{~h}$, which was followed by treatment with TNF- $\alpha$ for $24 \mathrm{~h}$. The results demonstrated that downregulation of PP4R1 significantly attenuated increased levels of glucose production following treatment with TNF- $\alpha$ (Fig. 4F), as well as PGC-1 $\alpha$, G6Pase and PEPCK mRNA and protein expression levels However, transfection of PP4R1 siRNA reversed the effect of TNF- $\alpha$ on the level of p-FOXO1/FOXO1 (Fig 4G and $\mathrm{H}$ ). In conclusion, these results suggested that miR-338-3p is involved in TNF- $\alpha$-mediated glucose production via targeting of PP4R1. 

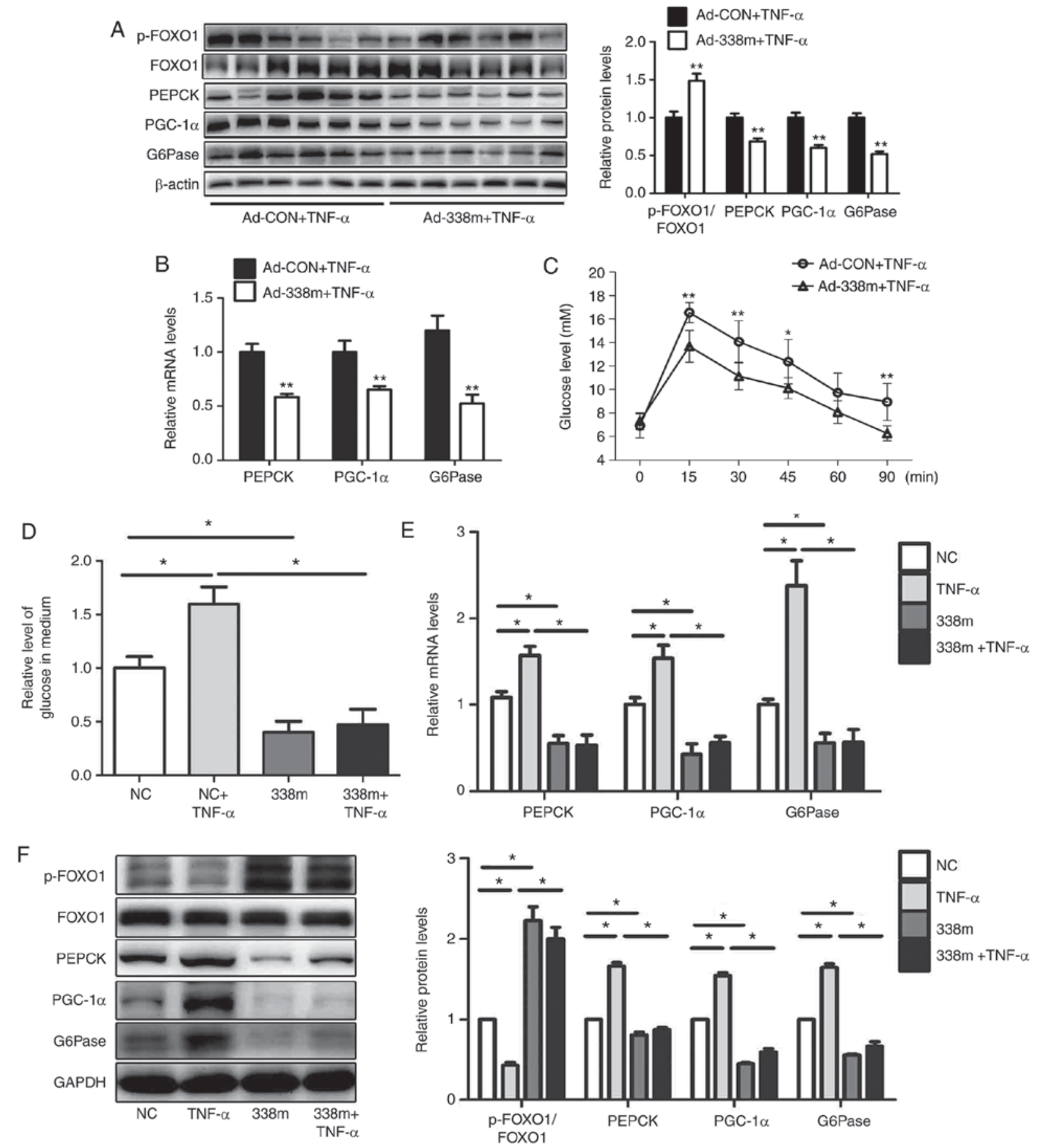

Figure 2. Overexpression of miR-338-3p attenuates TNF- $\alpha$-induced gluconeogenesis. Levels of p-FOXO1/FOXO1, PEPCK, PGC-1 $\alpha$ and G6Pase in the livers of TNF- $\alpha$-treated mice injected with Ad-338m via the tail vein for 7 days were analyzed by (A) western blot and (B) reverse transcription-quantitative polymerase chain reaction. (C) Pyruvate tolerance testing using mice was also performed. In cells, (D) relative levels of glucose in medium were determined, as well as (E) relative mRNA levels of PEPCK, PGC-1 $\alpha$ and G6Pase, and (F) protein levels of p-FOXO1/FOXO1, PEPCK, PGC-1 $\alpha$ and G6Pase, in TNF- $\alpha$-treated HEPA1-6 cells transfected with miR-338-3p mimics. Data are presented as the mean \pm standard error of the mean. For animal experiments, 6 mice were used. For cell experiments, $\mathrm{n}=4$ independent experiments. ${ }^{*} \mathrm{P}<0.05$ and ${ }^{* *} \mathrm{P}<0.01$ vs. control or as indicated. miR, microRNA; $\mathrm{p}-$, phosphorylated; FOXO1, forkhead box O1; PEPCK, phosphoenolpyruvate carboxykinase; PGC-1 $\alpha$, peroxisome proliferator-activated receptor $\gamma$ coactivator-1 $\alpha$; G6Pase, glucose-6-phosphatase; Ad, adenovirus; CON, control; TNF- $\alpha$, tumor necrosis factor- $\alpha$; $338 \mathrm{~m}$, miR-338-3p mimic; NC, mimic negative control.

\section{Discussion}

Chronic inflammation is a major factor resulting in the development of pathogenesis associated with insulin resistance (16). In addition, TNF- $\alpha$ promotes inflammation and attenuates insulin resistance in hepatocytes. Furthermore, in vivo and in vitro studies have suggested that treatment with
TNF- $\alpha$ blocks the insulin signalling pathway and results in insulin resistance (13).

In our previous study, it was demonstrated that treatment with TNF- $\alpha$ decreased glycogen synthesis and decreased the level of miR-338-3p in hepatocytes (13). It has been previously suggested that miR-338-3p has an important role in TNF- $\alpha$-mediated glycogenesis via regulation of PP4R1 (13). 

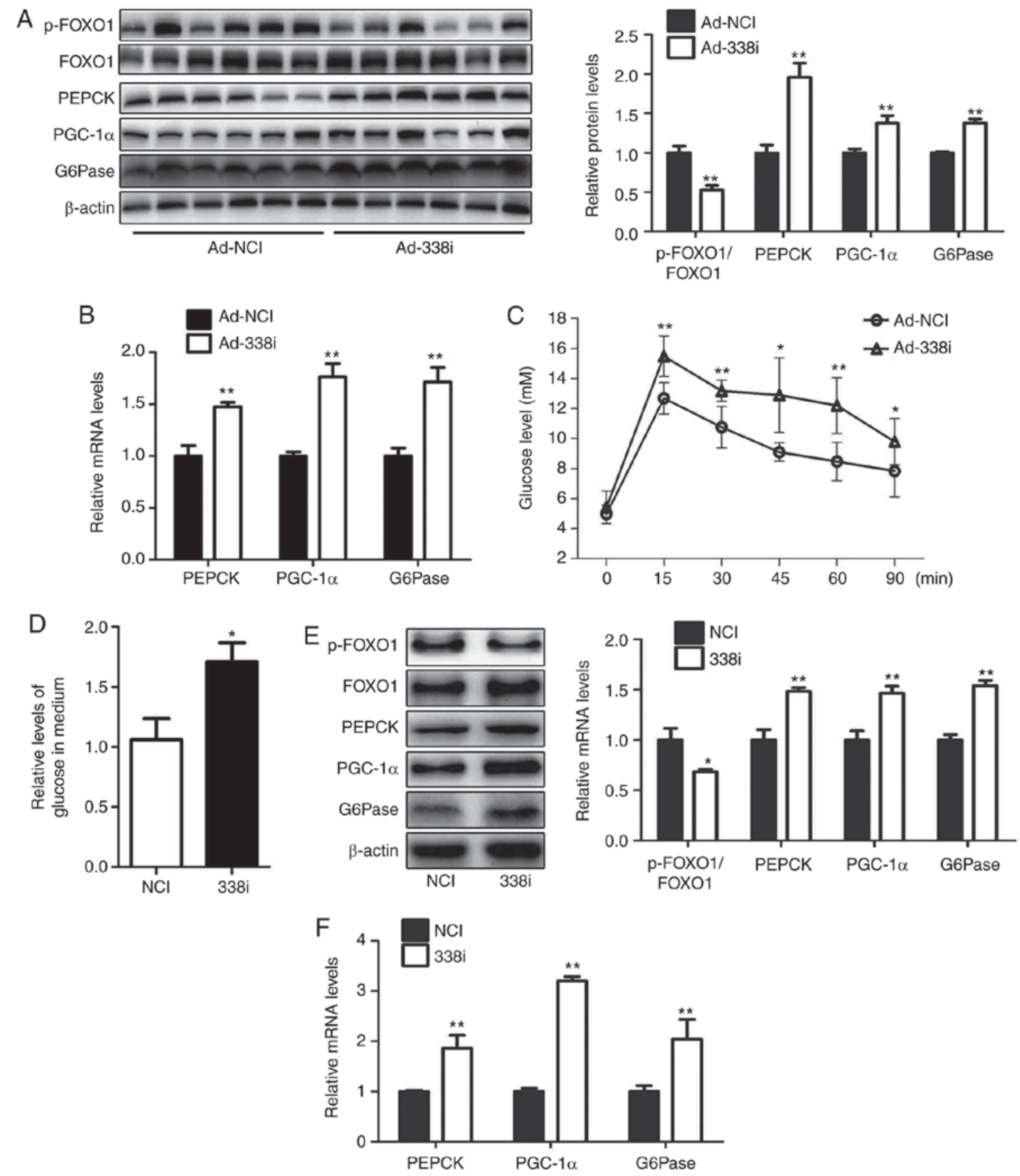

Figure 3. Downregulation of miR-338-3p enhances gluconeogenesis in hepatocytes. Levels of p-FOXO1/FOXO1, PEPCK, PGC-1 $\alpha$ and G6Pase in the livers of mice injected with Ad-338i via the tail vein for 7 days were analyzed by (A) western blot and (B) reverse transcription-quantitative polymerase chain reaction. (C) Pyruvate tolerance testing using mice was performed. (D) Relative levels of glucose in culture medium were also determined as well as (E) levels of p-FOXO1, FOXO1, PEPCK, PGC-1 $\alpha$ and G6Pase protein, and (F) PEPCK, PGC-1 $\alpha$ and G6Pase mRNA in HEPA1-6 cells transfected with miR-338-3p inhibitors were determined. Data are presented as the mean \pm standard error. For animal experiments, 6 mice were used. For cell experiments, $n=4$ independent experiments. "P<0.05 and ${ }^{* *} \mathrm{P}<0.01$ vs. control. miR, microRNA; p-, phosphorylated; FOXO1, forkhead box O1; PEPCK, phosphoenolpyruvate carboxykinase; PGC-1 $\alpha$, peroxisome proliferator-activated receptor $\gamma$ coactivator-1 $\alpha$; G6Pase, glucose-6-phosphatase; Ad, adenovirus; NCI, negative control inhibitor; $338 \mathrm{i}$, miR-338-3p inhibitor.

However, the effect of miR-338-3p on TNF- $\alpha$-mediated gluconeogenesis remains unknown. In the present study, whether miR-338-3p modulates gluconeogenesis via regulation of PP4R1 expression was investigated. In our previous study, we validated the effect of miR-338-3p mimics, miR-338-3p inhibitor, Ad-338m, Ad-338i and PP4R1-targeting siRNA in HEPA1-6 cells (13). Additionally, hepatic miR-338-3p level was increased $>10$ fold at 7 days after the Ad-338m injection; hepatic miR-338-3p level was decreased to $\sim 30 \%$ at
7 days after the AD-338i injection. The results revealed that treatment with TNF- $\alpha$ significantly induced gluconeogenesis in mouse liver and HEPA1-6 cells. In addition, it was demonstrated that miR-338-3p overexpression significantly suppressed glucose production in the livers of TNF- $\alpha$-treated mice and TNF- $\alpha$-treated HEPA1- 6 cells. Furthermore, the results revealed that downregulation of miR-338-3p induced gluconeogenesis in the livers of TNF- $\alpha$-treated mice and TNF- $\alpha$-treated HEPA1- 6 cells. Finally, it was demonstrated 

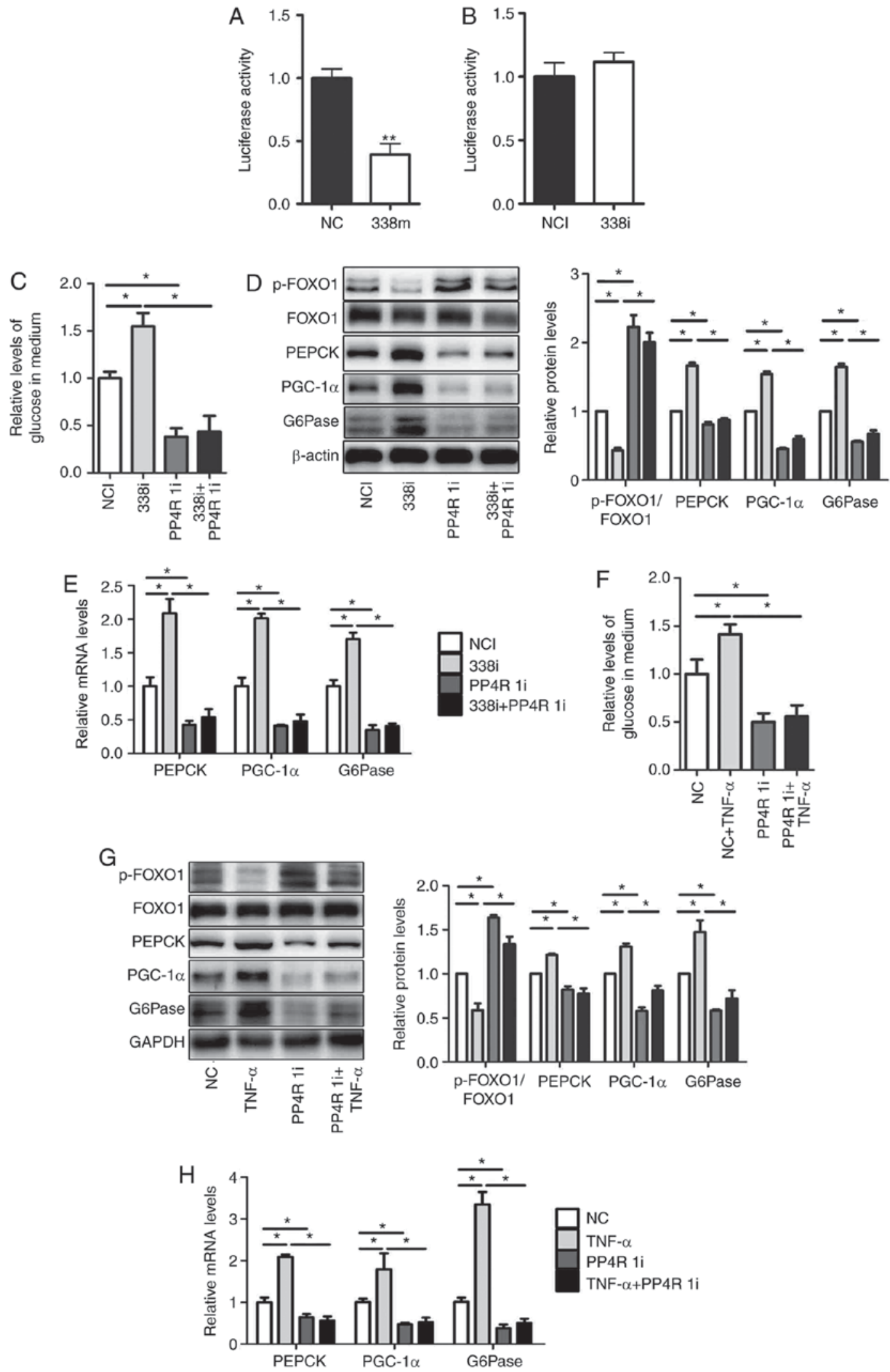

Figure 4. miR-338-3p is involved in TNF- $\alpha$-mediated gluconeogenesis via targeting of PP4R1. PP4R1 is a direct target of miR-338-3p. Luciferase activity was analyzed in HEPA1-6 cells transfected with (A) miR-338 mimics (B) or inhibitors. (C) Relative levels of glucose in medium, as well as (D) levels of p-FOXO1/FOXO1, PEPCK, PGC-1 $\alpha$ and G6Pase protein, and (E) PEPCK, PGC-1 $\alpha$ and G6Pase mRNA were determined in HEPA1-6 cells co-transfected with miR-338-3p inhibitors and PP4R1 small interfering RNA. (F) Relative levels of glucose in medium, as well as (G) protein levels of p-FOXO1/FOXO1, PEPCK, PGC-1 $\alpha$ and G6Pase, and (H) PEPCK, PGC-1 $\alpha$ and G6Pase mRNA were determined in HEPA1-6 cells treated with $10 \mathrm{ng} / \mathrm{ml} \mathrm{TNF-} \alpha$ for $24 \mathrm{~h}$ followed by transfection with PP4R1 small interfering RNA for $48 \mathrm{~h}$. Data are presented as the mean \pm standard error of the mean. Experiments were repeated four times. ${ }^{*} \mathrm{P}<0.05$ and ${ }^{* *} \mathrm{P}<0.01$ vs. control or as indicated. miR, microRNA; NC, mimic negative control; 338m, miR-338-3p mimic; NCI, negative control inhibitor; 338i, miR-338-3p inhibitor; PPR41, protein phosphatase 4 regulatory subunit 1; PP4R 1i, PP4R siRNA; p-, phosphorylated-; FOXO1, forkhead box O1; PEPCK, phosphoenolpyruvate carboxykinase; PGC-1 $\alpha$, peroxisome proliferator-activated receptor $\gamma$ coactivator-1 $\alpha$; G6Pase, glucose-6-phosphatase; NC, negative siRNA control; siRNA, small interfering RNA; TNF- $\alpha$, tumor necrosis factor- $\alpha$. 
that miR-338-3p was involved in TNF- $\alpha$-mediated glucose production via targeting of PP4R1.

Hepatic insulin resistance leads to increased gluconeogenesis in the liver, which may impair glucose metabolism homeostasis (17). miRs are a cluster of small, non-coding RNAs. Numerous miRs have important roles in the regulation of the activation of the insulin signalling pathway, including miR-291b-3p (18), miR-200s (19), miR-152 (20), miR-20a-5p (21), miR-19a (22) and miR-301a (23). The results of our previous study suggested that miR-338-3p regulates the AKT/GSK pathway via targeting of PP4R1, which subsequently regulates protein phosphatase 4 (PP4) expression (11). The miR-338-3p gene is located within intron 8 of the apoptosis-associated tyrosine kinase gene (24). In the present study, it was demonstrated that miR-338-3p modulates gluconeogenesis via regulation of AKT/FOXO1 pathway activation. Gluconeogenesis is an important metabolic pathway for the conversion of non-carbohydrate metabolites (lactate, glycerol and amino acids) to glucose, which provides energy for other organs during a prolonged fast; gluconeogenesis predominantly occurs in the liver and is regulated by insulin (25). Gluconeogenesis is regulated by two key enzymes in the liver: PEPCK and G6Pase. Hepatic expression of PEPCK and G6Pase is inhibited by insulin in the fed state (26). In the present study, it was revealed that hepatic overexpression of $\mathrm{miR}-338-3 \mathrm{p}$ induces the insulin signalling pathway, which suppresses PEPCK and G6Pase expression levels in gluconeogenesis both in vivo and in vitro.

The mechanism underlying the miR-338-3p role in TNF- $\alpha$-induced gluconeogenesis was also investigated in the present study. FOXO1 co-activates PGC1 $\alpha$, which subsequently induces PEPCK and G6Pase transcription. FOXO1 is the predominant modulator of insulin activity (27). Insulin suppresses FOXO1 activity via activation of the PI3K/AKT signalling pathway. Activated AKT phosphorylates FOXO1, which leads to nuclear exclusion of FOXO and subsequent suppression of FOXO-induced transcription (9). Furthermore, PGC1 $\alpha$ is an additional transcriptional co-activator that is activated under fasting conditions (28). Insulin suppresses PGC1 $\alpha$ activity via increased phosphorylation at Ser570 by activated AKT. In addition, the results of the present study revealed that miR-338-3p regulates FOXO1 phosphorylation via the PI3K/AKT pathway.

In a previous study, PP4R1 was revealed to be a direct target of miR-338-3p, which regulates AKT/glycogen synthase kinase pathway activation. PP4R1 is a regulatory subunit of PP4. PP4 has been previously reported to be an important regulator of TNF- $\alpha$-induced hepatic insulin resistance (12). In the present study, it was demonstrated that PP4R1 was involved in miR-338-3p-mediated gluconeogenesis.

In conclusion, the results of the present study suggested that miR-338-3p is involved in TNF- $\alpha$-induced hepatic gluconeogenesis in vivo and in vitro. miR-338-3p may be associated with $\mathrm{TNF}-\alpha$-induced gluconeogenesis via targeting of PP4R1.

\section{Acknowledgements}

Not applicable.

\section{Funding}

The present study was supported by the National Natural Science Foundation of China (grant nos. 81570789, 81600618 and 81700709), the Beijing Natural Science Foundation (grant no. 7182144) and the Beijing Hospital Nova Project (grant no. BJ-2018-138).

\section{Availability of data and materials}

The datasets used and/or analyzed during the current study are available from the corresponding author on reasonable request.

\section{Authors' contributions}

SW, LL, XC and XH conducted the cell experiments, JL and XS performed the animal experiments, $\mathrm{YZ}$ and TS constructed the luciferase reporter vector, JG, YM and WT performed pyruvate tolerance test and glucose production assay; LD and JL made contributions to the design of the present study. All authors read and approved the final manuscript.

\section{Ethics approval and consent to participate}

The present study was approved by Ethic and Animal Welfare committee College of Life Science Beijing Normal University.

\section{Patient consent for publication}

Not applicable.

\section{Competing interests}

The authors declare that they have no competing interests.

\section{References}

1. Shaw JE, Sicree RA and Zimmet PZ: Global estimates of the prevalence of diabetes for 2010 and 2030. Diabetes Res Clin Pract 87: 4-14, 2010.

2. Gaggini M, Morelli M, Buzzigoli E, Defronzo RA and Gastaldelli A: Non-alcoholic fatty liver disease (NAFLD) and its connection with insulin resistance, dislipidemia, atherosclerosis and coronary heart disease. Nutrients 5: 1544-1560, 2013.

3. Puig-Domingo $M$ and Pellitero S: New therapies for type 2 diabetes mellitus. Med Clin (Barc) 144: 560-565, 2015 (In Spanish).

4. Erol A: Insulin resistance is an evolutionarily conserved physiological mechanism at the cellular level for protection against increased oxidative stress. Bioessays 29: 811-818, 2007.

5. Nordie RC, Foster JD and Lange AJ: Regulation of glucose production by the liver. Annu Rev Nutr 19: 379-406, 1999.

6. Leclercq IA, Da Silva Morais A, Schroyen B, Van Hul N and Geerts A: Insulin resistance in hepatocytes and sinusoidal liver cells: mechanisms and consequences. J Hepatol 47: 142-156, 2007.

7. Bugianesi E, McCullough AJ and Marchesini G: Insulin resistance: A metabolic pathway to chronic liver disease. Hepatology 42: 987-1000, 2005.

8. Lu M, Wan M, Leavens KF, Chu Q, Monks BR, Fernandez S, Ahima RS, Ueki K, Kahn CR and Birnbaum MJ: Insulin regulates liver metabolism in vivo in the abesence of hepatic akt and foxo1. Nat Med 18: 388-395, 2012.

9. Yoon JC, Puigserver P, Chen G, Donovan J, Wu Z, Rhee J, Adelmant G, Stafford J, Kahn CR, Granner DK, et al: Control of hepatic gluconeogenesis through the transcriptional coactivator PGC-1. Nature 413: 131-138, 2001. 
10. Bartel DP: MicroRNAs: Genomics, biogenesis, mechanism, and function. Cell 116: 281-297, 2004.

11. Ono K: MicroRNA links obesity and impaired glucose metabolism. Cell Res 21: 864-866, 2011.

12. Zhao H, Huang X, Jiao J, Zhang H, Liu J, Qin W, Meng X, Shen T, Lin Y, Chu J and Li J: Protein phosphatase 4 (PP4) functions as a critical regulator in tumor necrosis factor (TNF)- $\alpha$-induced hepatic insulin resistance. Sci Rep 5: 18093, 2015.

13. Dou L, Wang S, Sun L, Huang X, Zhang Y, Shen T, Guo J, Man Y, Tang W and Li J: Mir-338-3p mediates Tnf-A-Induced hepatic insulin resistance by targeting PP4r1 to regulate PP4 expression. Cell Physiol Biochem 41: 2419-2431, 2017.

14. National Research Council (US) committee for the update of the Guide for the Care and Use of Labortory Animals: Guide for the care and use of laboratory animals, 8th edition. Washington (DC) Nation Academies Press, 2011.

15. Livak KJ and Schmittgen TD: Analysis of relative gene expression data using real-time quantitative PCR and the 2(-Delta Delta C(T)) method. Mehtods 25: 402-408, 2001.

16. Kim JH, Bachmann RA and Chen J: Interleukin-6 and insulin resistance. Vitam Horm 80: 613-633, 2009.

17. Fernandez-Valverde SL, Taft RJ and Mattick JS: MicroRNAs in $\beta$-cell biology, insulin resistance, diabetes and its complications. Diabetes 60: 1825-1831, 2011.

18. Guo J, Dou L, Meng X, Chen Z, Yang W, Fang W, Yang C, Huang X, Tang W, Yang J and Li J: Hepatic MiR-291b-3p mediated glucose metabolism by directly targeting p65 to upregulate PTEN expression. Sci Rep 7: 39899, 2017.

19. Dou L, Zhao T, Wang L, Huang X, Jiao J, Gao D, Zhang H, Shen T, Man Y, Wang S and Li J: miR-200s contribute to interleukin-6 (IL-6)-induced insulin resistance in hepatocytes. J Biol Chem 288: 22596-22606, 2013.
20. Wang S, Wang L, Dou L, Guo J, Fang W, Li M, Meng X, Man Y, Shen T, Huang X and Li J: MicroRNA 152 regulates hepatic glycogenesis by targeting PTEN. FEBS J 283: 1935-1946, 2016.

21. Fang W, Guo J, Cao Y, Wang S, Pang C, Li M, Dou L, Man Y, Huang X, Shen T and Li J: MicroRNA-20a-5p contributes to hepatic glycogen synthesis through targeting p63 to regulate p53 and PTEN expression. J Cell Mol Med 20: 1467-1480, 2016.

22. Dou L, Meng X, Sui X, Wang S, Shen T, Huang X, Guo J, Fang W, Man Y, Xi J and Li J: MiR-19a regulates PTEN expression to mediate glycogen synthesis in hepatocytes. Sci Rep 5: 11602, 2015.

23. Dou L, Wang S, Sui X, Meng X, Shen T, Huang X, Guo J, Fang W, Man Y, Xi J and Li J: MiR-301a mediates the effect of IL-6 on the AKT/GSK pathway and hepatic glycogenesis by regulating PTEN expression. Cell Physiol Biochem 35: 1413-1424, 2015.

24. Jacovetti C, Jimenez V, Ayuso E, Laybutt R, Peyot ML, Prentki M, Bosch F and Regazzi R: Contribution of intronic mir-338-3p and its hosting gene AATK to compensatory beta-cell mass expansion. Mol Endocrinol 29: 693-702, 2015.

25. Radzuk J and Pye S: Hepatic glucose uptake, gluconeogenesis and the regulation of glycogen synthesis. Diabetes Metab Res Rev 17: 250-272, 2001.

26. Oh KJ, Han HS, Kim MJ and Koo SH: Transcriptional regulators of hepatic gluconeogenesis. Arch Pharm Res 36: 189-200, 2013.

27. Kousteni S: FoxO1, the transcriptional chief of staff of energy metabolism. Bone 50: 437-443, 2012.

28. Puigserver P, Rhee J, Donovan J, Walkey CJ, Yoon JC, Oriente F, Kitamura Y, Altomonte J, Dong H, Accili D and Spiegelman BM: Insulin-regulated hepatic gluconeogenesis through FOXO1-PGC-1alpha interaction. Nature 423: 550-555, 2003. 